\title{
An event-related visual occlusion method for examining anticipatory skill in natural interceptive tasks
}

\author{
DaVID L. MANN \\ University of New South Wales, Sydney, New South Wales, Australia \\ and Australian Institute of Sport, Bruce, Australian Capital Territory, Australia \\ BRUCE ABERNETHY \\ University of Hong Kong, Hong Kong, China \\ and University of Queensland, Brisbane, Queensland, Australia \\ AND \\ Damian Farrow, Mark DaVis, ANd Wayne Spratford \\ Australian Institute of Sport, Bruce, Australian Capital Territory, Australia
}

\begin{abstract}
This article describes a new automated method for the controlled occlusion of vision during natural tasks. The method permits the time course of the presence or absence of visual information to be linked to identifiable events within the task of interest. An example application is presented in which the method is used to examine the ability of cricket batsmen to pick up useful information from the prerelease movement patterns of the opposing bowler. Two key events, separated by a consistent within-action time lag, were identified in the cricket bowling action sequence - namely, the penultimate foot strike prior to ball release (Event 1), and the subsequent moment of ball release (Event 2). Force-plate registration of Event 1 was then used as a trigger to facilitate automated occlusion of vision using liquid crystal occlusion goggles at time points relative to Event 2. Validation demonstrated that, compared with existing approaches that are based on manual triggering, this method of occlusion permitted considerable gains in temporal precision and a reduction in the number of unusable trials. A more efficient and accurate protocol to examine anticipation is produced, while preserving the important natural coupling between perception and action.
\end{abstract}

Skilled performance in high-speed interceptive tasks relies on finely tuned perceptual-motor responses developed over many years of extensive practice. For interceptive sporting skills such as a return of serve in tennis or a goalkeeper saving a soccer penalty, every moment counts, and skilled athletes effectively "make time" via superior anticipation. This involves being more attuned to kinematic information provided by the opposition player's movements prior to ball flight (Abernethy \& Russell, 1987; Jones \& Miles, 1978). The skilled baseball batter, for example, can "read" the body movements of the pitcher to anticipate the type of pitch before the ball is released. This early information pickup may, in turn, facilitate earlier selection and initiation of the most appropriate hitting movement, consequently improving hitting performance (Shim, Carlton, Chow, \& Chae, 2005).

\section{Using Visual Occlusion to Measure \\ Anticipatory Skill}

Testing for skilled anticipation has predominantly used a temporal occlusion paradigm, in which the con- tribution to performance of visual information available before specific points in an event sequence is examined through occlusion of all subsequent visual information. The most common time point for occlusion in studies of anticipation in sport has occurred at the final moment immediately prior to the availability of confirmatory ballflight information - for example, the point of racquet-ball contact when one is receiving a tennis serve, or contact between foot and ball when one is keeping goal against a soccer penalty kick.

The temporal occlusion paradigm has relied heavily on the use of video display simulations to maintain experimental rigor and simplicity of administration (Abernethy, Thomas, \& Thomas, 1993). Participants typically view footage edited to occlude display information at critical points in the action sequence and, following occlusion, are required to predict the resultant ball direction (or other key characteristics) either verbally or using a pen-and-paper response. Video displays, although methodologically convenient, are limited when one is seeking to accurately

D. L. Mann, d.mann@unsw.edu.au 
simulate the full visual information inherent in a natural display. Some information, such as three-dimensional information aiding depth perception, is inevitably either lost or diminished in most video simulations. The temporal occlusion paradigm can be applied in situ using liquid crystal goggles that are capable of transitions between transparency and opacity within $5 \mathrm{msec}$ (Milgram, 1987). This provides an important advance in efforts to ensure that the visual information that is presented more accurately replicates that experienced in the natural environment. Studies examining expert anticipation in situ using liquid crystal goggles have usually reproduced the expert advantage that has been noted in laboratory studies using video simulations (e.g., Abernethy, Gill, Parks, \& Packer, 2001; Farrow, Abernethy, \& Jackson, 2005; Müller \& Abernethy, 2006; Müller et al., 2009; Starkes, Edwards, Dissanayake, \& Dunn, 1995).

Rather than relying on verbal or pen-and-paper responses, a further improvement in the design for numerous studies of anticipation has been to preserve the natural coupling between perception and action by more accurately representing the actual movements produced in the performance environment. Early attempts to allow for this did so by using simplified movements that were performed when observing video-based simulations (e.g., Savelsbergh, Williams, van der Kamp, \& Ward, 2002; Williams \& Davids, 1998). Examinations of anticipation using protocols that require naturally coupled movement responses are desirable, though, since verbal and/or simplified motor responses may be unable to elicit responses via the same visual-motor pathways relied on in situ. For example, a response stimulus providing an opportunity for target interception is more likely to accurately test the vision-for-action pathway that is relied on in the performance setting (Króliczak, Heard, Goodale, \& Gregory, 2006; Mann, Abernethy, \& Farrow, in press-a; van der Kamp, Rivas, van Doorn, \& Savelsbergh, 2008). More recently, in situ study designs have allowed more natural movements to occur, providing the opportunity for interception between an object and the target (Farrow \& Abernethy, 2003; Müller \& Abernethy, 2006; Shim et al., 2005). Farrow and Abernethy (2003) used an in situ design to demonstrate that observation of the full magnitude of the expert advantage requires a movement that maintains the natural coupling between perception and action rather than a verbal response. Wherever possible, experimental designs should replicate the perception and action requirements of the performance environment as closely as is permissible in order to permit a full picture of expertise to be gained.

\section{Challenges in Using Occluding Goggles to Examine Anticipation in Situ}

Although in situ studies more closely simulate the naturally occurring performance conditions, most are currently limited by an inability to accurately occlude vision at specified times in natural actions like hitting, throwing, and bowling. Video-based simulations are able to occlude vision within one frame (typically $33-40 \mathrm{msec}$ ) of any specific point of interest. In situ occlusion studies, in con- trast-in which event-related occlusions are achieved by an experimenter pressing a button to manually estimate the trigger point for occlusion - typically accept time windows of up to $300 \mathrm{msec}$ (e.g., Farrow \& Abernethy, 2002; Müller \& Abernethy, 2006; Müller et al., 2009). High-speed video footage has been used to perform post hoc analysis of occlusion times in an effort to overcome such a limitation, ensuring that only those trials meeting inclusion criteria are considered for analysis. A consequence of this method is that larger trial numbers are typically conducted to compensate for those to be discarded, and this creates a particular problem in terms of the workload requirements of the actor(s) performing the action to which the participant is responding. In order to enhance stimulus reproducibility across participants, it is desirable to have the same actor (or at least only a small set of actors) viewed by each of the participants; this results in high actor workloads, even without the issue of trial loss or duplication resulting from errors in administration of the occlusion condition. Clearly, a method to more accurately occlude vision in situ is desirable, and this necessitates the development of a more precise eventrelated trigger for the occlusion points of interest.

Oudejans and Coolen (2003) occluded the vision of basketball free-throw shooters by using an online assessment of kinematic changes, improving on the buttonpress approach. Although this technique presents an adaptable and potentially accurate means of occlusion, it relies heavily on extensive knowledge of computer programming for algorithm development to detect movement patterns, and on the use of expensive and specialized equipment. A simple and accurate means of occlusion is desirable to provide an alternate approach to advance on currently available methods for the application of occlusion methods in situ.

This article outlines the development of a new method for accurate, event-related occlusion of vision in situ. A four-step process was followed. First, we identified a typical interceptive task from a high-speed sport (the task of batting, in cricket) for which the in situ measurement of anticipatory skill using a temporal occlusion method has been deemed desirable. Second, we examined the event sequence within this task to identify a measurable, repeatable event (Event 1) that preceded the event of interest for occlusion (Event 2). The temporal lag between these two events was measured, and the consistency of this lag was verified both within and between individuals. Third, an automated system was developed for the controlled occlusion for time points relative to Event 2, on the basis of triggering from Event 1. Finally, the moment of occlusion relative to Event 2 was validated, with the results compared with those for the manual triggering methods reported previously in the literature.

\section{METHOD}

The four-step process took place as follows:

\section{Anticipation in Cricket Batting As a Test Case}

An abundance of time-stressed interceptive tasks exist for which the early pickup of visual information is understood to facilitate performance; we chose the task of cricket batting, in which batters attempt to hit a ball projected toward them by an opposition bowler 
at velocities that can exceed $150 \mathrm{kph}$. Batters must overcome severe time constraints in order to be successful, with as little as $450 \mathrm{msec}$ of ball flight available for the execution of a successful response. Proficiency in anticipation of ball flight characteristics can aid in overcoming these demands; the earlier these features are identified, the more time there is available to carry out a response (Abernethy, 1981). Early information pickup can make possible bat-ball contact that may not have occurred otherwise; or, more subtly, it may enable earlier body positioning, which may, in turn, permit a more aggressive (and hence more effective) bat-ball contact (Mann, Abernethy, $\&$ Farrow, in press-b). In laboratory-based temporal occlusion studies using video displays, skilled cricket batters have been shown to be better than novices at anticipating delivery characteristics prior to the ball's being released by a bowler (Abernethy \& Russell, 1984; Müller, Abernethy, \& Farrow, 2006; Penrose \& Roach, 1995). In situ studies using occluding goggles (e.g., Müller \& Abernethy, 2006; Müller et al., 2009) have confirmed this advantage for expert batsmen. The in situ examination of anticipation in cricket batting is an important methodological advance, since it recognizes the essential perception-action coupling that is embedded in skilled movements (Farrow \& Abernethy, 2003) and affords a consideration of the additional importance that early ball-flight information may have for expertise in batting (Land \& McLeod, 2000; Müller \& Abernethy, 2006; Müller et al., 2009).

Cricket bowling provides a suitably reliable movement sequence with deterministic kinematics to help specify particular characteristics in advance of ball flight. The bowler can produce different deliveries with very distinct characteristics (e.g., ball direction, ball swing), which require their own distinct kinematic nuances, providing skilled batters sensitive to these with an opportunity to pick up identifiable advance information. In the bowling action sequence (Figure 1), a bowler runs toward the batter and jumps into a final delivery stride prior to ball release. In this stride, the (back) foot farthest from the batter lands first, followed by the leading ( front) foot, each landing with considerable peak vertical ground reaction forces (front foot $=2.37 \times$ body weight; back foot $=5.75 \times$ body weight; Hurrion, Dyson, \& Hale, 2000). The landing of each foot precedes the moment of ball release. This reliable sequence of events, plus the extent of the foot-ground interaction, presents the potential for the automated triggering of occluding goggles.

\section{Identification of a Measurable Preocclusion Event}

Occlusion points in studies of anticipation skill are generally designed and expressed relative to a single critical event: For cricket bowling, this is characteristically the moment of ball release. Oc- clusion points immediately prior to ball release are of interest to examine how early predictions about ball type and direction can be made purely on the basis of the bowler's prerelease movement pattern, whereas those after release help one examine whether skilled players are better able to make use of early ball-flight information. To facilitate automated occlusion at set times either before or after the moment of ball release (Event 2), a preceding event in the bowling sequence (Event 1) must be identified, and it must fulfill three primary requirements. First, Event 1 must occur sufficiently prior to Event 2 to provide time to trigger occlusions before Event 2, taking into account both delays in detecting Event 1 and any inherent delays in the automated system. Second, Event 1 must be reliably recordable in order to register the trigger point. Third, the time lag between Events 1 and 2 must be highly repeatable to ensure that the first event accurately predicts the second.

Back-foot contact (BFC; see Figure 2) presents promise as a reliable trigger point that addresses the aforementioned requirements for time points relative to ball release. First, BFC (as a potential Event 1) always occurs prior to ball release (Event 2). On initial inspection, it appears to occur sufficiently far in advance of Event 2 to allow essential computation and occlusion triggering to occur, but sufficiently close in time to offer the prospect of minimizing errors that are based on extrapolation of the time lag. Second, registration of the considerable reaction forces generated by contact with the ground offers a potential means for registering the onset of Event 1. In an effort to address the third criterion, an analysis was undertaken to assess whether the interevent time lag is sufficiently repeatable to permit the time of ball release (Event 2) to be predicted accurately from the time of BFC (Event 1).

The interevent time lag between $\mathrm{BFC}$ and ball release was determined by visual inspection of recorded video footage. High-speed video records (Phantom v4.2, Vision Research), filmed at 100 $250 \mathrm{~Hz}$ from a side-on perspective, were sourced for 32 bowlers of different ages and skill levels. BFC (Event 1) was defined as the first frame in which the back foot (in the final footfall prior to ball release) made contact with the ground, and ball release (Event 2) was the first frame where separation between the bowler's hand and the ball could be identified. The interevent time lag calculated across a series of trials (see Table 1) examined both the absolute degree of variability within this lag and whether this was influenced by either the bowler's skill level or performance conditions (game vs. laboratory). A highly repeatable time lag was observed for 5 elite bowlers recorded in a laboratory, with an average standard deviation of 9 msec (range 8-11 msec; intraclass correlation of mean $R=.97$ ). Comparable findings were observed for state representative bowl-
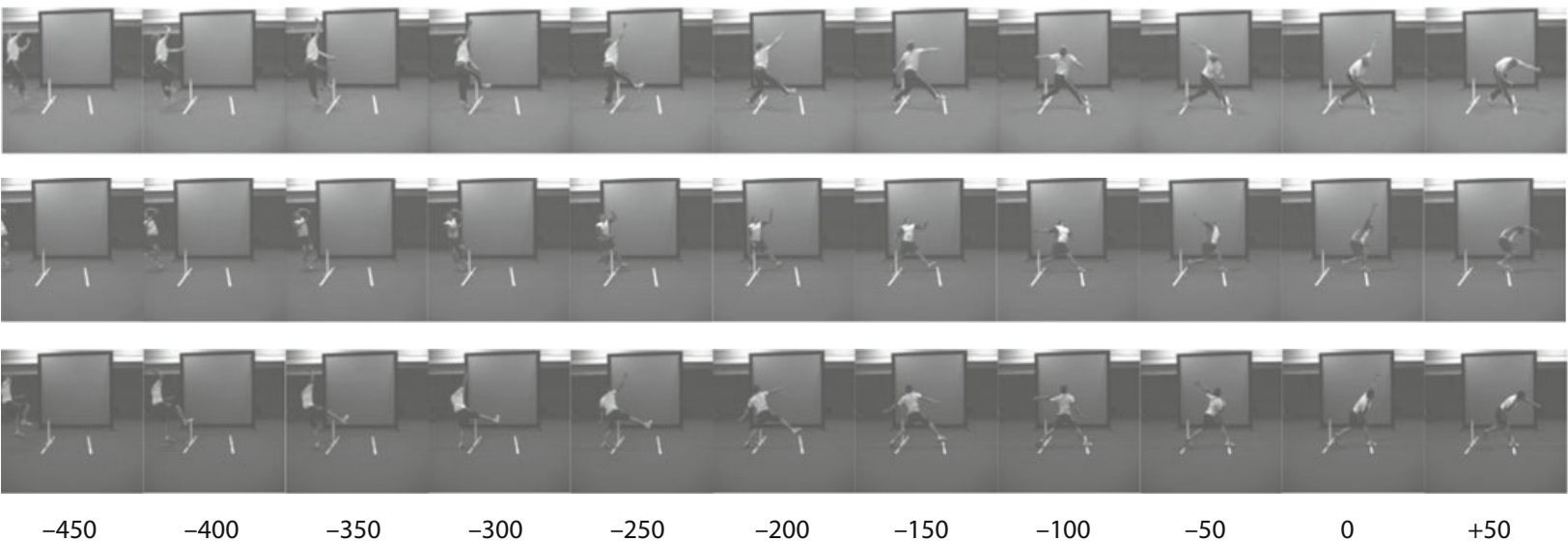

Time Relative to Ball Release (msec)

Figure 1. Time course of delivery stride for three exemplar cricket bowling action sequences. 


\section{A Back-Foot Contact}

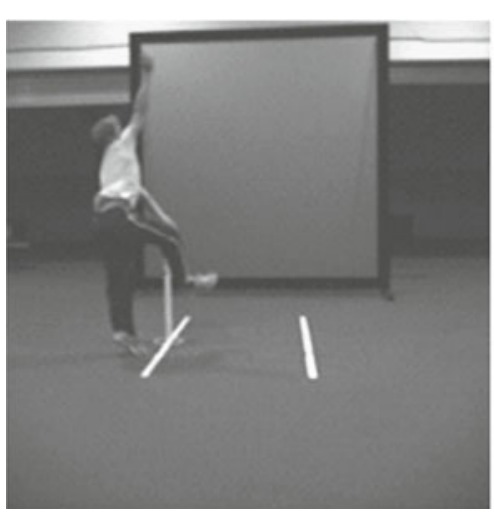

B Ball Release

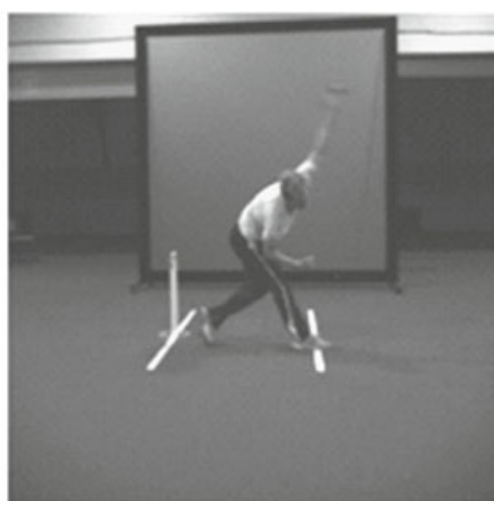

Figure 2. Illustration of the moment of (A) back-foot contact and (B) ball release in a cricket bowling action sequence.

ers recorded in a laboratory $(S D=11 \mathrm{msec}$; range $=6-16 \mathrm{msec}$; $R=.88)$, under-19 elite bowlers recorded in a laboratory $(S D=$ $11 \mathrm{msec}$; range $=5-17 \mathrm{msec} ; R=.92)$, and for elite bowlers recorded in a match $(S D=12 \mathrm{msec}$; range $=11-12 \mathrm{msec} ; R=.97)$. Furthermore, the consistency found in experts was also observed for less-skilled bowlers; footage of six local club-level bowlers revealed interevent time lags of similar magnitude and consistency $(S D=$ $9 \mathrm{msec}$; range $=7-13 \mathrm{msec} ; R=.96$ ).

These results demonstrate that although there are individual differences in the mean time lag for BFC-ball release, there is a high degree of consistency within bowlers regardless of skill level or testing environment. Furthermore, Event 1 across this sample occurred at least $200 \mathrm{msec}$, on average, prior to Event 2, ensuring a reliable trigger point for occlusion that occurs sufficiently in advance of ball release to permit reliable automated occlusions to be made relative to the time of ball release (Event 2).

\section{Development of an Automatically Triggered Occlusion Approach}

The identification of a temporally consistent interevent latency made possible the development of an automated system for occlusion. This permitted the visual occlusion of response stimuli at time points relative to Event 2, on the basis of triggering from Event 1. In the section that follows, we describe the three essential components of the automated system: the registration of BFC; the introduction of a bowler-specific time lag; and the method of automated triggering.

Registration of BFC. A force plate was used to register the onset of ground reaction forces generated by BFC. The size $(900 \times$
$600 \mathrm{~mm}$ ) and location (situated where the back foot of most cricket bowlers would land) of the force plate ensured that bowlers with conventional bowling approaches would not be required to make a conscious effort to land on the plate. Signals from ground-embedded force plates (Kistler 9287BA, Kistler Instrumente AG, Switzerland) were relayed to a specifically designed comparator box to detect the onset of force. ${ }^{1}$ The $z$ direction voltage generated by the force plate was compared with a set point controlled by a potentiometer; the manual dial on the potentiometer was adjusted to the lowest possible setting above threshold, so that the onset of the smallest permissible force on the plate would generate a voltage to exceed the set point. When this occurred, a transistor-transistor logic (TTL) signal was generated. This signal was relayed via a 25 -pin parallel cable connection, and it provided an external trigger to a laptop running ToTaL control (v2.0.1, Translucent Technologies, Canada) - the software designed specifically to control the occlusion of PLATO goggles (PLATO Model P-1, Translucent Technologies, Canada). The signal generated by the ToTaL control program was transmitted via a modular four-connector phone jack to both the PLATO occlusion goggles ${ }^{2}$ and to a triple LED (see Figure 3 ). The ToTaL control program ensures occlusion by switching the PLATO goggles from transparent to opaque. It does so either immediately on receiving a signal, or following a predetermined, manually entered time delay. Illumination of the LEDs occurred contemporaneously with the occlusion of vision, facilitating post hoc analysis of video footage to verify occlusion time relative to ball release.

Introduction of a bowler-specific time for signal delay. A bowler-specific time for signal delay was calculated using the mean

Table 1

Characteristics of Interevent Time Lag (in Milliseconds) Across Skill Levels and Testing Environments

\begin{tabular}{|c|c|c|c|c|c|c|c|c|}
\hline \multirow[b]{2}{*}{ Skill Level } & \multirow{2}{*}{$\begin{array}{l}\text { Frame Rate } \\
\text { (Frames } \\
\text { perSecond) }\end{array}$} & \multirow{2}{*}{$\begin{array}{c}\text { Number of } \\
\text { Bowlers }\end{array}$} & \multirow{2}{*}{$\begin{array}{l}\text { Trials per } \\
\text { Bowler }\end{array}$} & \multicolumn{2}{|c|}{$M$} & \multicolumn{2}{|c|}{$S D$} & \multirow{2}{*}{$\begin{array}{c}\text { Intraclass } \\
\text { Correlation } \\
\text { of Mean }\end{array}$} \\
\hline & & & & Value & Min-Max & Value & Min-Max & \\
\hline \multicolumn{9}{|c|}{ Laboratory Environment* } \\
\hline Elite & 250 & 5 & 30 & 327 & $287-405$ & 9 & $7-10$ & .97 \\
\hline State & 250 & 9 & 30 & 296 & $258-337$ & 11 & $6-16$ & .88 \\
\hline Elite under-19 & 250 & 10 & 30 & 286 & 209-334 & 11 & $5-17$ & .92 \\
\hline Club & 200 & 6 & 10 & 354 & $298-405$ & 9 & $7-13$ & .96 \\
\hline \multicolumn{9}{|c|}{ Game Environment } \\
\hline Elite & 100 & 2 & 15 & 361 & $314-407$ & 12 & $11-12$ & .97 \\
\hline
\end{tabular}

*Back-foot contact for these trials was recorded as the point at which the back foot was flat on the ground, typically $20 \mathrm{msec}$ after initial contact with the ground. 


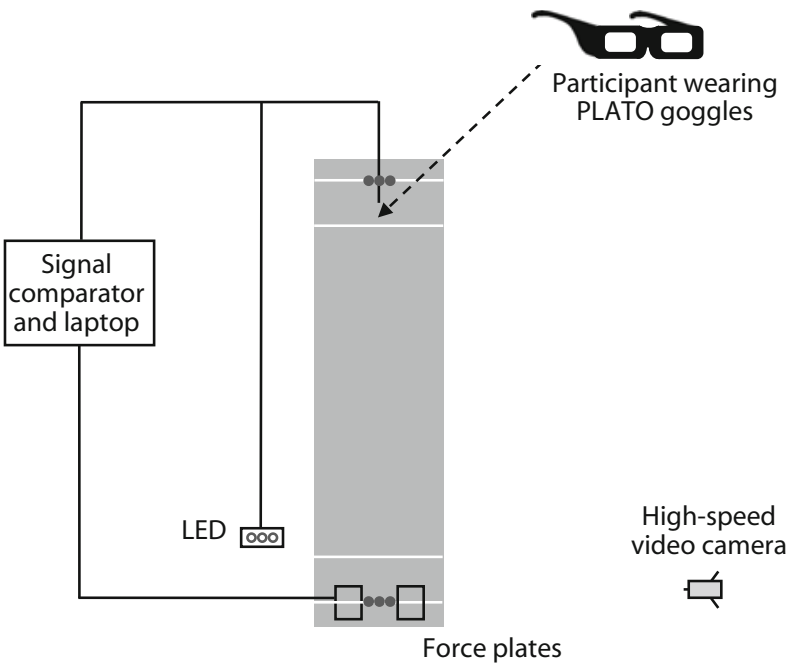

X Starting position of bowler

Figure 3. Schematic diagram of the experimental setup employed for in situ occlusion.

interevent (BFC-ball release) time lag to facilitate occlusion at, or for time points relative to, ball release. High-speed video footage of bowlers was recorded from a side-on perspective so that (1) the moment of BFC, (2) the moment of ball release, and (3) the LED were all visible within the field of view. The minimal signal delay setting was entered into the ToTaL control software program to ensure that the LED would be illuminated on registration of BFC. The LED was used to establish BFC in order to discount any potential disparity between the visual and force-plate registration of BFC, and to allow for any inherent delay in the automated system because of signal processing and transmission.

Programming the software to trigger occlusion at the mean interevent time lag would result in ball flight being visible for at least $50 \%$ of trials. To occlude vision immediately prior to ball release, the signal delay was shortened by 2 standard deviations of the mean interevent time lag. Statistically, this ensured that almost $98 \%$ of trials occluded prior to ball release, with minimal chance for the presentation of any ball-flight information. As a result, the bowlerspecific signal delay for occlusions immediately prior to ball release was calculated according to the following formula:

$$
\text { Signal delay }=\text { Mean }_{\text {time lag }}-2 S D_{\text {time lag. }} \text {. }
$$

Automated triggering of occlusion goggles. The bowlerspecific signal delay-corresponding to both the bowler and the desired time of occlusion-was entered into the ToTaL control software program prior to each trial. As a result, an appropriately delayed signal was relayed to the occlusion goggles and the LED following BFC

\section{Validation of the Automated Method}

Three skilled male cricket bowlers (mean age $=29.1 \pm 6.1$ years; mean weight $=76 \pm 7 \mathrm{~kg}$ ) were recruited to take part in a validation of the automated system of occlusion. All were considered to be medium-paced bowlers $(85-110 \mathrm{kph})$ and had played in the first or second grade of their regional club cricket competition within the previous 12 months.

The first step in the validation was to establish the interevent signal delay for each of the 3 bowlers. High-speed video footage $(500 \mathrm{~Hz})$ was recorded from a side-on perspective, with the minimum permissible signal delay entered into the $\mathrm{ToTaL}$ control software program to illuminate the LED upon registration of BFC. Video footage was recorded for 10 trials from each of the 3 bowlers, and it was subsequently viewed to record Event 1 (BFC; moment of LED illumination) and Event 2 (ball release; first frame where separation between the ball and the hand was visible). On the basis of the interevent time lag (Bowler 1, $287 \pm 11 \mathrm{msec}$; Bowler 2, 313 $\pm 9 \mathrm{msec}$; Bowler 3, 406 $\pm 5 \mathrm{msec}$ ), the appropriate signal delay was calculated for each bowler, with the intention of occluding vision at, or immediately prior to, ball release.

The second step in the validation was to establish the moment that occlusion took place relative to ball release. To this end, each bowler delivered a further 20 trials with his specific signal delay entered into the ToTaL control software program. High-speed video footage $(500 \mathrm{~Hz})$ was used to calculate for each trial the instance of occlusion (moment of LED illumination) relative to the point of ball release.

\section{RESULTS}

Table 2 demonstrates the results for the point of occlusion relative to Event 2 for each of the 3 bowlers. The moment of occlusion occurred either prior to or at ball release for 58 out of 60 trials, with the earliest occlusion occurring $50 \mathrm{msec}$ prior to ball release. Occlusion transpired $2 \mathrm{msec}$ after ball release for the two trials that did not occlude prior to Event 2. On average, the moment of occlusion occurred between 11 and $23 \mathrm{msec}$ prior to ball release (depending on the bowler).

\section{DISCUSSION}

The automated system presented here allows for the accurate and relatively simple occlusion of vision in situ at specific points in the action sequence. The accurate measurement of interevent time lag ensures that, for the vast majority of trials, occlusion occurs within a small and well-defined time period preceding ball release. Accurate measurement of the bowler-specific mean (and $S D$ ) interevent time lag ensures that occlusion, theoretically, occurs on average at a time point 2 standard deviations prior to ball release. Accordingly, almost $98 \%$ of trials occlude within 4 standard deviations of ball release, a window approximating $40 \mathrm{msec}$ in the wide sample of bowlers presented here. This is comparable to the one-frame limit achievable with conventional video simulations ( $33-40 \mathrm{msec})$, and it is very favorable when compared with the $300-\mathrm{msec}$ windows reported in previous in situ studies (Farrow \& Abernethy, 2003; Müller \& Abernethy, 2006).

Table 2

Validation Data for Moment of Occlusion Relative to Ball Release

\begin{tabular}{|c|c|c|c|c|c|}
\hline \multirow[b]{2}{*}{ Bowler } & \multicolumn{4}{|c|}{$\begin{array}{c}\text { Time Delay (msec) From Ball } \\
\text { Release to Occlusion }\end{array}$} & \multirow{2}{*}{$\begin{array}{c}\text { Number of } \\
\text { Trials That } \\
\text { Occluded } \\
\text { After Ball } \\
\text { Release }\end{array}$} \\
\hline & $M$ & $S D$ & Min & Max & \\
\hline 1 & -23 & 14 & -50 & -10 & 0 \\
\hline 2 & -11 & 10 & -34 & 2 & 2 \\
\hline 3 & -14 & 6 & -28 & -6 & 0 \\
\hline
\end{tabular}

Note-Negative value indicates that visual occlusion occurred prior to ball release. 
The temporal accuracy of this method (outlined on a theoretical basis) has been validated using high-speed footage to examine the moment of occlusion relative to ball release for 3 examplar bowlers. These results demonstrated that occlusion took place prior to (or at) ball release for $97 \%$ of trials, a figure close to the $98 \%$ expected when applying the outlined method. There was strong congruence between the theoretically anticipated time of occlusion prior to Event 2 (on the basis of mean occlusion taking place 2 standard deviations prior) and the empirically measured time delay (Bowler 1, 22 vs. $23 \mathrm{msec}$; Bowler 2, 18 vs. $11 \mathrm{msec}$; Bowler 3, 10 vs. $14 \mathrm{msec}$, respectively). Occlusions took place across all 3 bowlers within a $52-\mathrm{msec}$ window, which is comparable to the 33-40 msec achievable with video simulations and is certainly favorable when compared with the $300 \mathrm{msec}$ currently accepted by in situ studies relying on a manual buttonpress for occlusion.

The enhanced temporal precision for occlusion control outlined in this article facilitates a more powerful experimental design when examining anticipation in situ. With occlusion transpiring within a small window of time, this greater control ensures that fewer trials need to be excluded from analysis as a result of falling outside of the desired time range. This results either in less trials being necessary overall for the required number of trials to be included for analysis, or in potentially greater statistical power for the same number of trials performed. Furthermore, if an in situ protocol such as those used previously accepts large time windows for occlusion, participant decisions are likely to be made on the basis of a wide variety of occlusion times that are assumed to be uniform. For example, an occlusion that occurred $250 \mathrm{msec}$ prior to ball release will be grouped in the same category with one that occluded immediately prior to ball release, but it will deny important visual information that transpired between occlusion and ball release. The greater precision made possible with this new technique ensures the availability of as much information as possible on all trials.

Rather than being limited to only the sport of cricket, this protocol may be used for other actions, such as a volleyball serve or a rugby sidestep in which the opposing player moves toward the observer. The reliance of such a protocol on force plates need not be limiting, since the approach may be just as effective using cheaper and similarly simple triggering mechanisms such as pressure pads or light gates, for appropriate action sequences.

When seeking to examine action sequences in which the opposing player is more stationary (e.g., tennis serve or baseball pitch), it may be possible to map the time course of the ground reaction force to discover events that are highly correlated with the critical point in the sequence. Rather than relying on the onset of loading, this may depend on reaching a desired threshold force for an accurate trigger. For example, the back-foot weight transference of a tennis serve or the unloading from the back foot of a baseball pitcher might occur at a highly reliable time prior to ball contact or release, providing a potential signalling point for accurate occlusion that is comparable to that provided by $\mathrm{BFC}$ in the cricket bowling case.

\section{CONCLUSIONS}

A more accurate and reliable means of event-related visual occlusion allows for enhanced precision in the examination of perceptual expertise. Rather than relying on the observation of video simulations, in situ designs are advantageous in that they preserve the skilled performer's highly developed links between perception and action. The automated approach we have described enhances considerably the precision with which event-related display occlusions can be made, affording a more sensitive method and making possible more powerful experimental designs. Furthermore, the protocol is relatively fast and simple to execute. Such an advance allows us to build more readily on our existing understanding of the development of skill in interceptive actions.

\section{AUTHOR NOTE}

Correspondence concerning this article should be addressed to D. L. Mann, School of Optometry and Vision Science, University of New South Wales, Sydney, NSW 2052, Australia (e-mail: d.mann@unsw .edu.au).

\section{REFERENCES}

Abernethy, B. (1981). Mechanisms of skill in cricket batting. Australian Journal of Sports Medicine, 13, 3-10.

Abernethy, B., Gill, D. P., Parks, S. L., \& Packer, S. T. (2001). Expertise and the perception of kinematic and situational probability information. Perception, 30, 233-252. doi:10.1068/p2872

Abernethy, B., \& Russell, D. G. (1984). Advanced cue utilization by skilled cricket batsmen. Australian Journal of Science \& Medicine in Sport, 16, 2-10.

Abernethy, B., \& Russell, D. G. (1987). Expert-novice differences in an applied selective attention task. Journal of Sport Psychology, 9, 326-345.

Abernethy, B., Thomas, K. T., \& Thomas, J. T. (1993). Strategies for improving understanding of motor expertise (or mistakes we have made and things we have learned!!). In J. L. Starkes \& F. Allard (Eds.), Cognitive issues in motor expertise (pp. 317-356). Amsterdam: North-Holland.

Farrow, D., \& ABernethy, B. (2002). Can anticipatory skills be learned through implicit video-based perceptual training? Journal of Sports Sciences, 20, 471-485.

FARRow, D., \& ABERnethy, B. (2003). Do expertise and the degree of perception-action coupling affect natural anticipatory performance? Perception, 32, 1127-1139. doi:10.1068/p3323

Farrow, D., Abernethy, B., \& Jackson, R. C. (2005). Probing expert anticipation with the temporal occlusion paradigm: Experimental investigations of some methodological issues. Motor Control, 9, 332351.

Hurrion, P. D., Dyson, R., \& HaLe, T. (2000). Simultaneous measurement of back and front foot ground reaction forces during the same delivery stride of the fast-medium bowler. Journal of Sports Sciences, 18, 993-997.

Jones, C. M., \& MiLes, T. R. (1978). Use of advanced cues in predicting the flight of a lawn tennis ball. Journal of Human Movement Studies, 4, 231-235.

Króliczak, G., Heard, P., Goodale, M. A., \& Gregory, R. L. (2006). Dissociation of perception and action unmasked by the hollowface illusion. Brain Research, 1080, 9-16. doi:10.1016/j.brainres .2005.01.107

Land, M. F., \& McLeod, P. (2000). From eye movements to actions: How batsmen hit the ball. Nature Neuroscience, 3, 1340-1345.

Mann, D. L., Abernethy, B., \& Farrow, D. (in press-a). Action specificity increases anticipatory performance and the expert advantage in natural interceptive tasks. Acta Psychologica. doi:10.1016/j.actpsy .2010 .04 .006 . 
Mann, D. L., Abernethy, B., \& Farrow, D. (in press-b). The resilience of natural interceptive actions to refractive blur. Human Movement Science. doi:10.1016/j.humov.2010.02.007

Milgram, P. (1987). A spectacle-mounted liquid-crystal tachistoscope. Behavior Research Methods, Instruments, \& Computers, 19, 449456.

Müller, S., \& ABernethy, B. (2006). Batting with occluded vision: An in situ examination of the information pickup and interceptive skills of high- and low-skilled cricket batsmen. Journal of Science \& Medicine in Sport, 9, 446-458. doi:10.1016/j.jsams.2006.03.029

Müller, S., Abernethy, B., \& Farrow, D. (2006). How do worldclass cricket batsmen anticipate a bowler's intention? Quarterly Journal of Experimental Psychology, 59A, 2162-2186. doi:10.1080/02643290600576595

Müller, S., Abernethy, B., Reece, J., Rose, M., Eid, M., MCBEAN, R., ET AL. (2009). An in-situ examination of the timing of information pickup for interception by cricket batsmen of different skill levels. Psychology of Sport \& Exercise, 10, 644-652. doi:10.1016/j .psychsport.2009.04.002

Oudejans, R. R. D., \& Coolen, B. H. (2003). Human kinematics and event control: On-line movement registration as a means for experimental manipulation. Journal of Sports Sciences, 21, 567-576. doi:10.1080/0264041031000101917

Penrose, J. M. T., \& Roach, N. K. (1995). Decision making and advanced cue utilization by cricket batsmen. Journal of Human Movement Studies, 29, 199-218.

Savelsbergh, G. J. P., Williams, A. M., VAn der Kamp, J., \& Ward, P.
(2002). Visual search, anticipation and expertise in soccer goalkeepers. Journal of Sports Sciences, 20, 279-287.

Shim, J., Carlton, L. G., Chow, J. W., \& Chae, W.-S. (2005). The use of anticipatory visual cues by highly skilled tennis players. Journal of Motor Behavior, 37, 164-175. doi:10.3200/JMBR.37.2.164-175

Starkes, J. L., Edwards, P., Dissanayake, P., \& Dunn, T. (1995). A new technology and field test of advance cue usage in volleyball. $R e$ search Quarterly for Exercise \& Sport, 66, 162-167.

VAN DER Kamp, J., Rivas, F., van Doorn, H., \& Savelsbergh, G. [J. P.] (2008). Ventral and dorsal system contributions to visual anticipation in fast ball sports. International Journal of Sport Psychology, 39, 100-130.

Williams, A. M., \& Davids, K. (1998). Visual search strategy, selective attention, and expertise in soccer. Research Quarterly for Exercise \& Sport, 69, 111-128.

\section{NOTES}

1. The circuit diagram is available from the corresponding author on request.

2. The occlusion goggles were tethered in this paradigm, but they did not restrict participant movements. Modifications permitting wireless occlusion currently present considerable time delays, and future models are anticipated to address this limitation.

(Manuscript received June 19, 2009; revision accepted for publication December 13, 2009.) 